\title{
ADGenKinetics: An Algorithmically Differentiated Library for Biochemical Networks Modeling via Simplified Kinetics Formats
}

\author{
Atiyah Elsheikh \\ Austrian Institute of Technology, Vienna, Austria
}

\begin{abstract}
This work demonstrates the compact but powerful freely available Modelica library ADGenKinetics for descriptive modeling of biochemical reaction networks using simplified kinetics formats. While existing powerful works and guidelines for modeling biochemical reaction networks based on classical mechanistic kinetics already exist, in this work a first attempt of utilizing the power of Modelica constructs for providing a compact implementation of simplified kinetic formats with generalized structured formulas is presented. This gives the opportunity of realizing biochemical reaction networks using few number of reaction components, in contrast to libraries based on classical mechanistic kinetics which require hundreds of reaction components. Moreover, ADGenKinetics is the first algorithmically differentiated Modelica library that is enhanced with differentiated components by which parameter sensitivities are additionally computed with minimal efforts from the user perspective.

Keywords: enzyme kinetics, biochemical reaction networks, systems biology, algorithmic differentiation
\end{abstract}

\section{Introduction}

Modelica as a universal modeling language with a lot of capabilities for supporting hierarchical modeling, multidisciplinary modeling, object-oriented reusable components and different modeling flavours with a large degree of freedom and creativity is continuously attracting the attention of many scientific fields. However, in the field of Systems Biology aiming at studying cellular process with the aid of mathematical models, there are still few published non-standardized attempts for modeling biochemical reaction networks for describing the metabolism within cellular activities, one of the core modeling activities demanded by many applications of the field of Systems Biology.
This work demonstrates a comprehensive overview of the compact Modelica library ADGenKinetics for a specific set of reaction kinetics. These subsets of enzyme kinetics are referred to as simplified kinetics formats and are represented by generalized structured kinetics formulas suitable for biochemical reactions with arbitrary number of substrates, products, inhibitors and activators. The employment of generalized kinetics have two advantages from two perspectives:

1. From the modeling perspective: Utilization of generalized kinetics formulas provides the opportunity of implementing a compact library with so few numbers of components that the user neither needs to choose an enzyme kinetic component from a long list of components nor needs to self implement newer enzyme kinetics for newer cases of non considered reactions

2. From the implementation perspective: By efficient employment of powerful Modelica language constructs, the implementation of highly specialized practical library for modeling biochemical network applications gets simplified.

The proposed library is distinguished by the following criteria:

- It is suitably adequate to get linked with specialized graphical editors for modeling biochemical reaction networks and for other applications of automatic model generation

- It is the first algorithmically differentiated library by which algorithmic differentiation (AD) techniques $[6,11]$ are directly applied at the library level [5]. The resulting additional subpackage contains extended components in which parameter sensitivities, i.e. derivatives of model variables w.r.t. model parameters, are represented. 
- it is open-source and provided under the Modelica License 2.

The rest of the work is structured as follows: section 2 presents a quick introduction to biochemical networks modeling. Section 3 gives rather a quick overview of various classical and simplified kinetic laws but comprehensive enough for appreciating this contribution. Section 4 demonstrates the proposed library, its advanantages and limitations along an example in section 5. Finally, outlook is given in section 6.

\section{Background and Terminology}

\subsection{Modeling biochemical reaction networks}

Biochemical reaction network models are used for describing the dynamics of molecular species and their interaction within the cellular metabolism [9]. Usually such models are based on the continuum ${ }^{1}$ and homogeneity assumptions ${ }^{2}$. The law of mass conservation ${ }^{3}$ is used for describing the rate of change in the mass of intermediate metabolites (i.e. biochemical substances) in a biochemical reaction network. The resulting models typically have the following structure:

$$
\dot{c}=N \cdot v(c, \alpha), \quad c(0)=c_{0}
$$

where $c \in R^{m}$ stands for vectors of the metabolite concentrations, $v=v(c, p) \in R^{n}$ is a vector of reaction rates described by enzyme kinetics, $\alpha$ is kinetic parameters vector describing enzyme characteristics and $N \in R^{m \times n}$ is the reactions stoichiometry describing the number of participating molecules in any single reaction [15].

Figure 1 demonstrates a typical biochemical network of enzymatic reactions termed as the Spirallus which represents an abstraction of Tri-Carboxylic Acid (TCA) cycle [14]. The set of freely distributed metabolites $A, B, C, D, E, F$ are viewed as nodes, while the reactions are viewed as intermediate edges among the metabolites [2]. With the presence of substrates being taken up through the initial reaction $v_{u p t}$, intermediate reactions become active and the two products $E_{e x}, F_{e x}$ get produced as long as enough substrate molecules are taken up. Some of the reactions are irreversible such as $v_{3}$ (i.e. the flow of materials is conceptually only in the forward direction) while others

\footnotetext{
${ }^{1}$ All chemical species involved have such a high copy number to be described by a continuous concentration value

${ }^{2}$ Diffusion processes are so fast that concentrations can be considered to be spatially homogeneous

${ }^{3}$ the mass within a closed system remains constant over time
}

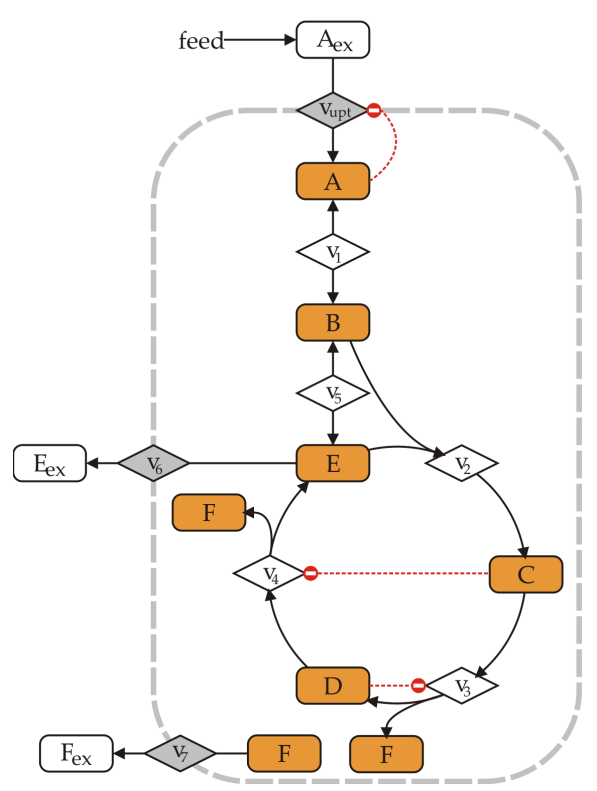

Figure 1: Spirallus: An Abstraction of the TCA cycle

are reversible such as $v_{1}$. The reactions $v_{\text {upt }}, v_{3}, v_{4}$ are inhibited by the molecules of the metabolites $A, D, C$ acting as inhibitors respectively. Analogously, specific molecules of metabolites may act as activators by accelerating certain reactions. Inhibitors and activators are referred to as effectors or modifiers. A mathematical model for describing the process dynamics is given as follows:

$$
\begin{aligned}
{[\dot{A}] } & =v_{\text {upt }}-v_{1} & {[\dot{B}] } & =v_{1}-v_{2}-v_{5} \\
{[\dot{C}] } & =v_{2}-v_{3} & {[\dot{D}] } & =v_{3}-v_{4} \\
{[\dot{E}] } & =v_{4}+v_{5}-v_{2}-v_{6} & {[\dot{F}] } & =v_{3}+v_{4}-v_{7}
\end{aligned}
$$

The state variables $A, B, . ., F_{e x}$ corresponds to the concentration of metabolites. The algebraic variables $v_{j}$ describe the reaction rates via algebraic functions referred to as enzyme kinetics, the topic of the following section.

\section{Enzyme kinetics}

Vital cellular processes at the metabolism level are performed according to the present set of enzymatic reactions networks. The base elements of such networks are the involved enzymatic reactions. For instance, within an uni-uni enzymatic reaction in the form:

$$
S+E \stackrel{k_{1}}{\underset{k_{-1}}{\rightleftharpoons}} \quad E S \quad \stackrel{k_{2}}{\longrightarrow} \quad E+P
$$


the molecules of the specific enzyme $E$ binds with the molecules of the substrate $S$ according to a rate constant $k_{1}$. Similarly, $k_{-1}$ is a rate constant describing the decomposition rate of the complex $E S$ into $E$ and $S$. The resulting enzyme-substrate complex ES molecules are vastly transformed to the product $P$. The reaction rate $v$ of such transformation, i.e. product formation, is modeled by enzyme kinetics. Such kinetics typically correspond to nonlinear functions of the following form:

$$
v(t)=e \cdot f(c(t), \alpha)
$$

where $e$ is the amount of the associated enzyme, $\alpha$ a set of parameters corresponding to enzyme characteristics and $c(t)$ the concentration of the involved substrates, products and effectors [1]. In case of a reversible reaction, $f$ can be usually expressed in terms of forward and backward reaction rate as $v=v^{f w d}-$ $v^{b w d}$. In this case, the overall direction of the reaction is then the sign of $v$. Many enzyme kinetics approaches for describing the function $f$ exist some of which are demonstrated in the following subsections.

\subsection{Mechanistic kinetics}

In order to emphasize the importance of simplified kinetics formats, the widely used classical mechanistic kinetics are introduced as a motivation. Mechanistic kinetics describe the reaction rates of biochemical enzymatic reactions by involving the underlying enzyme binding mechanisms within the mathematical model. For instance, the simple reaction (2) is modelled by Michaelis-Menten kinetic. Its analytical derivation based on the quasi-steady state assumption (i.e. $k_{-1}, k_{1} \gg k_{2}$ ) leads to the following formula:

$$
v=\frac{k_{2}[E]_{0}[S]}{\frac{k_{-1}+k_{2}}{k_{1}}+[S]}=\frac{V_{\max }[S]}{K_{m}+[S]}
$$

The parameter $K_{m}$ corresponds to the substrate concentration that yields the half-maximal reaction rate $V_{\max } / 2$. These two parameters represent enzymatic characteristics demonstrating how quickly the enzyme becomes saturated and what its maximum activity is.

\section{Reactions with effectors}

For enzymatic activities influenced by effectors various types and binding mechanisms exist, cf. figure 2 for various inhibition mechanisms. Mechanistic kinetics distinguish such types of inhibitions mechanisms through their mathematical formulation according to whether

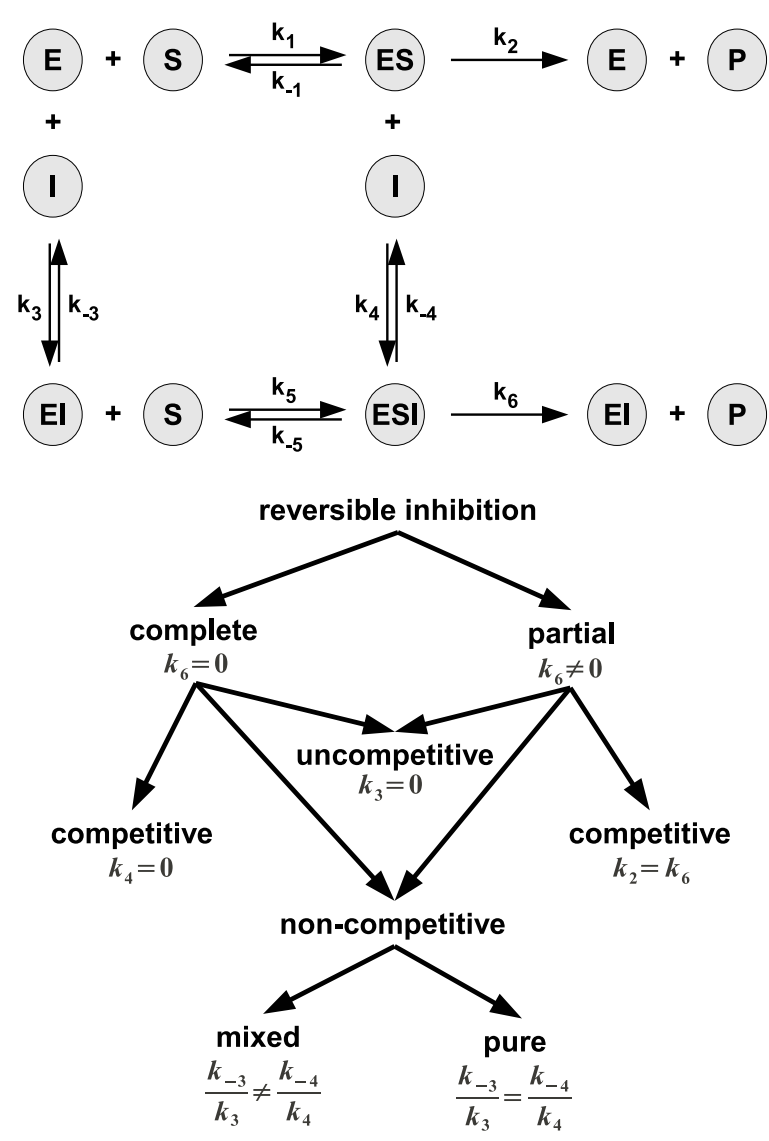

Figure 2: A summary of different types of inhibition mechanisms

- the inhibitor binds to the complex $E S$

- the inhibitor binds to $S$

- the reversibility of the inhibition

For instance, the analytical derivation of a mechanistic kinetic for an irreversible reaction inhibited by $I$ according to complete competitive inhibition leads to:

$$
v=\frac{V_{\max }[S]}{K_{m}\left(1+[I] / K_{I}\right)+[S]}
$$

Where $K_{I}$ is a parameter that expresses the ratio of $E I$ formation to $E I$ decomposition. Within competitive inhibition, the inhibitor $I$ competes with the substrate $S$ for binding with the enzyme $E$. In this case, the release of $P$ is blocked by $I$, cf. figure 2. Similar discussion regarding activation mechanisms leads to the fact that a wide range of mechanistic kinetics formulas exists distinguishing all these various mechanisms.

\section{Multi-substrate reactions}

For cellular reactions with more than one substrate and one product, very likely to arise in the cellular 
metabolism, mechanistic kinetics are more sophisticated. Their analytical derivation additionally considers the sequence in which substrates bind and products are released. For example, within a bi-bi reaction, (two substrates $S_{1}, S_{2}$ and two products $P_{1}, P_{2}$ ) the underlying enzymatic mechanisms are differentiated according to whether binding to enzyme is done

- in random order, (i.e. $E$ binds with both of $S_{1}$ and $S_{2}$ in any order)

- in a sequential order, (i.e. $S_{2}$ binds only with the complex $E S_{1}$ )

- in an alternate binding of substrates and release of products (ping-pong mechanisms)

as well as

- which intermediate complexes are formed (only $E S_{1}, E S_{2}$ or also $\left.E S_{1} S_{2}\right)$

- Interactions among reactants (e.g. inhibition through product formation)

For example, the kinetic of an ordered bi-bi reaction (i.e. binding in a specific order)

$$
\begin{aligned}
E+S_{1} \rightleftharpoons & E S_{1}+S_{2} \rightleftharpoons E S_{1} S_{2} \rightleftharpoons \\
& E P_{1} P_{2} \rightleftharpoons P_{1}+E P_{2} \rightleftharpoons E+P_{1}+P_{2}
\end{aligned}
$$

is described with the equation:

$$
v=\frac{V_{\max }\left[S_{1}\right]\left[S_{2}\right]}{K_{i S_{1}} K_{m S_{2}}+K_{m S_{2}}\left[S_{1}\right]+\left[S_{1}\right]\left[S_{2}\right]}
$$

In summary, each combination of assumptions regarding the underlying enzymatic reaction leads to a unique kinetic formula. This results in enormous number of possible equation patterns corresponding to combinatorially high number of different assumptions. Such equations do not necessarily follow a general equation pattern if they are expressed in terms of mechanistic parameters rather than elementary rate constants $k_{i}$. This causes some difficulties by modeling since hundreds of components need to be separately implemented for expressing different enzyme binding mechanisms.

\subsection{Generalized kinetics formats}

As already shown, mechanistic kinetics characterize detailed description of the underlying enzymatic mechanism. These kinetics pose however some problems when used for describing enzymatic reactions within cellular environment. Under such crowded conditions, a lot of effectors may influence the enzyme activity. When considering all typical interactions, the corresponding derived kinetic becomes very complex and parameter dependencies are enhanced when estimating the parameters with experimentally generated data leading to serious problems in the process of model identification [16].

This argument motivates the use of generalized kinetics which relay on more simplified assumptions two of which are introduced. The first type is the so-called convenience kinetics which assumes a reversible rapid equilibrium with random binding mechanism [10]. In this way, the corresponding kinetic of any reaction with arbitrary number of substrates $S_{i}$, products $P_{j}$, inhibitors $I_{b}$ and activators $A_{a}$ becomes:

$$
\begin{aligned}
v=\prod_{a} \frac{K_{A_{a}}+\left[A_{a}\right]}{K_{A_{a}}} \cdot \prod_{b} \frac{K_{I_{b}}}{K_{I_{b}}+\left[I_{b}\right]} & \\
& \frac{V_{\max }^{f w d} \prod_{i} \frac{\left[S_{i}\right]}{K_{m S_{i}}}-V_{\max }^{b w d} \prod_{j} \frac{\left[P_{j}\right]}{K_{m P_{j}}}}{\prod_{i}\left(1+\frac{\left[S_{i}\right]}{K_{m S_{i}}}\right)+\prod_{j}\left(1+\frac{\left[P_{j}\right]}{K_{m P_{j}}}\right)-1}
\end{aligned}
$$

Another kinetic format is the linlog kinetic [8] given by:

$$
\begin{aligned}
v=v^{0}+\sum_{i} \alpha_{i} \cdot \ln \left(\frac{S_{i}}{S_{i}^{0}}\right)+\sum_{j} \beta_{j} \cdot \ln \left(\frac{P_{j}}{P_{j}^{0}}\right)+ \\
\sum_{a} \gamma_{a} \cdot \ln \left(\frac{A_{a}}{A_{a}^{0}}\right)+\sum_{a} \delta_{b} \cdot \ln \left(\frac{I_{b}}{I_{b}^{0}}\right)
\end{aligned}
$$

In contrary to mechanistic parameters, which provide descriptive physical insights into enzymatic mechanisms, linlog parameters are based on scaled sensitivities describing the influence of characteristic changes of enzymes on a referenced reaction rate at a reference steady-state $v^{0}$.

One of the main advantages of the presented kinetics in the context of this work is that they are expressed in terms of generalized structured formulas very adequate for compact implementation and automatic generation of highly complex models. However, one of the limitation of such kinetics is that they may not describe the enzymatic behavior accurately in some boundary cases as shown in [7]. 


\section{Overview of the library}

\subsection{The Biochem Library}

For modeling biochemical networks with Modelica, considerable efforts have been realized by the Biochem library [12]. It provides the essential guidelines and design principles for achieving this goal, eg. basic implementable interfaces and basic types. According to the available publications, Biochem provides about 99 abstract reaction types under the restriction that a reaction can get connected to at most three substrates, three products and one effector. Out of these abstract types, many mechanistic reaction kinetics can be derived. Within the library Metabolic, a published implementation of Biochem, at least 180 kinetics are implemented and classified according to the number of substrates and products within many sub-packages. If all combination of reaction assumptions are considered, still many hundred of reaction kinetics need to be inserted. If more than one effector is considered, a realistic scenario for biochemical reaction networks in cellular environment, the number of required components corresponding to various kinetics would be so high.

\subsection{The ADGenKinetics Library}

In this work, a compact implementation of simplified kinetics is demonstrated following the main guidelines provided by Biochem w.r.t. the library structure, physical units, naming conventions and some of the implementation. The main differences appear whenever the mathematical structures of the simplified kinetics are utilized for implementing interfaces for the underlying generalized formulas. These interfaces are specialized according to the number of reactants, products, specific effectors, reversibility etc. By exploiting powerful Modelica constructs, realization of simplified kinetics require very few number of components out of which realistic biochemical networks are easily constructed, modeled and simulated. On the other hand classical mechanistic kinetics within implementation of Biochem requires a large number of components. Users are likely to insert additional kinetics whenever new or slightly modified biochemical reaction networks need to be modeled.

Figure 3 summarizes the presented library. The following packages are available:

- Interfaces: connectors, classification interfaces and icons

- NodeElements: components for nodes

- Reactions: components for reactions

- Derivatives: extended components for computing parameter sensitivities

- Examples: biochemical network models

Further two subpackages within NodeElements and Reactions exist corresponding to two ways of declaration of connectors within components:

1. dynamic: parametrized number of connections

2. static: fixed number of connections

The main differences of both ways and their advantages and disadvantages are emphasized in this section along with the given examples. Common interfaces and abstract classes are located above these packages.

\subsection{Connectors}

The fundamental laws on which biochemical reaction network models rely i.e. the continuum and homogeneity assumptions and the law of conservation (cf. section 2.1) translates into the terminology of Modelica as follows:

Listing 1: Implementation of chemical ports

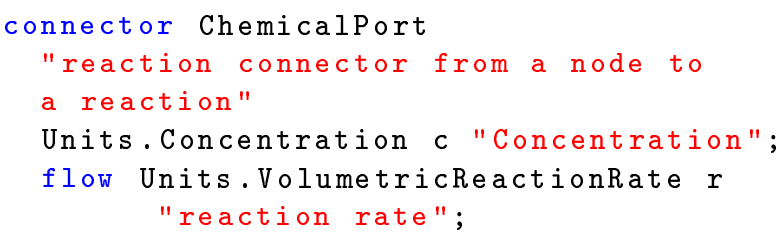

That is, the concentration of a substance is the potential variable while the flow rate of materials represents the flow variables when connecting nodes and reactions together. The mathematical models of biochemical reaction networks do not require a node to distinguish between a connection from an ingoing reaction and a connection to an outgoing reaction. The sign of the reaction rate is explicitly determining whether the considered node is a substrate or a product of the connected reaction.

This situation is different with connections to nodes from a reaction side. The kinetic formula distinguishes between a substrate node and a product node, cf. equation (8). Consequently additional connectors, ChemicalPort_S, ChemicalPort_P extending the connector 


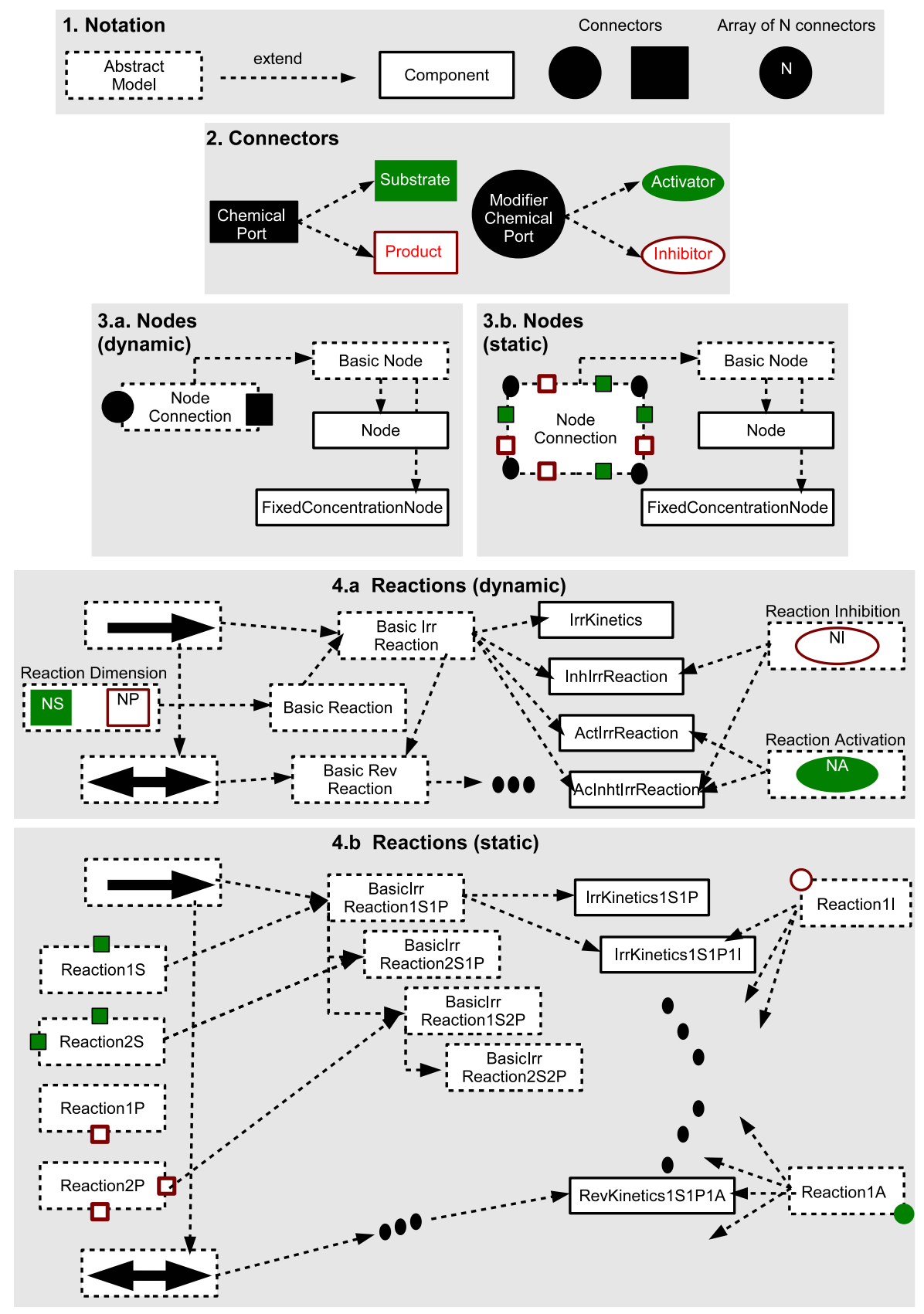

Figure 3: An overview of the library with static/dynamic number of connections

ChemicalPort with distinguished icons for differentiating between connections to substrates and connections to products are considered. Additionally, the specialized connector ModifierChemicalPort between effectors and reactions is provided. This connector includes only the concentration of the respective node. Similarly two icons are provided to distinguish activators from inhibitors.

\subsection{Nodes}

In Biochem, implementation of nodes is realized at three levels of abstraction:

1. NodeConnections: A class providing the basic interfaces and icons, about 8 connectors as a reactant and 4 connectors as a modifier

2. BasicNode: An abstract class realizing basic implementation of nodes and extending NodeConnections

3. Node: An implementation of BasicNode describ- 
ing the concentration dynamics

The static subpackage is directly taken from Biochem.

In the subpackage dynamic, only one connector for reactants and one connector for modifiers [15] is given as follows:

Listing 2: Implementation of node connections

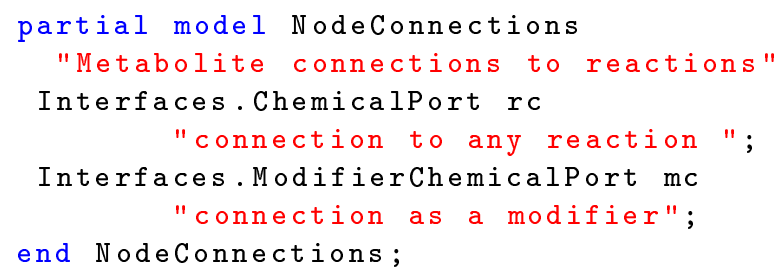

The abstract class BasicNode in dynamic looks as follows:

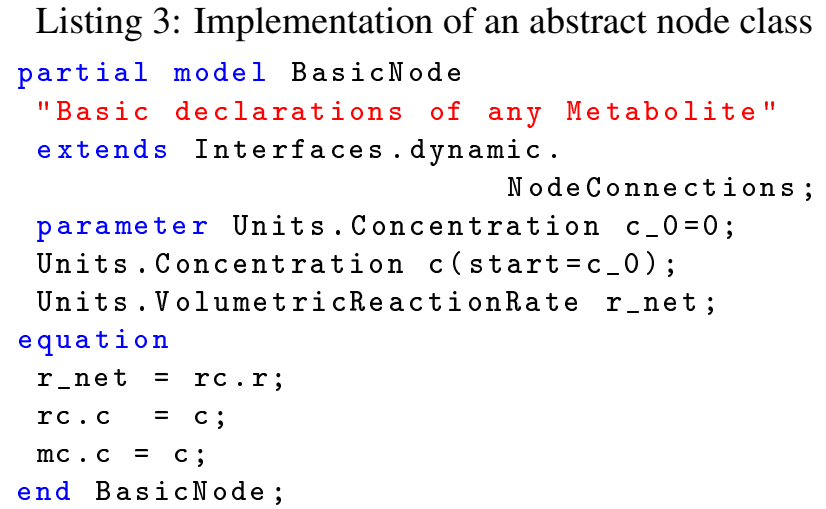

Listing 3: Implementation of an abstract node class

Direct implementation of BasicNode is realized in the models FixedConcentrationNode and Node:

Listing 4: Implementation of a node

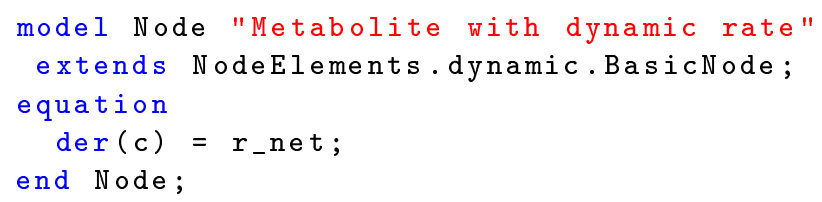

Further types of nodes exist in Biochem.

\subsection{Reactions}

Each generalized kinetic format is realized within a subpackage. Currently, the subpackage convenience is implemented. The realization of other simplified kinetic formats like linlog kinetics is analogously straightforward.

\subsection{1 dynamic}

In this subpackage, convenience kinetics are implemented by extending several abstract classes which specifies a reaction according to:
1. its dimension: how many substrates and products are involved as well as the stoichiometry of the reactants

2. its reversibility

3. whether the reaction is effected by other modifiers, how many and their types

The implementation of these basic classes are shown as follows:

Listing 5: The dimension of a reaction

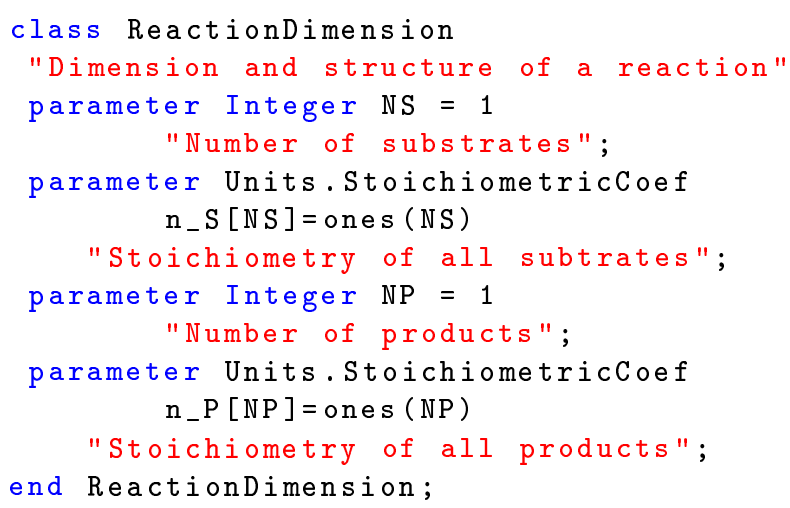

Using the previous class, an abstract type for reactions slightly modified version from the one provided in Biochemis given as follows:

Listing 6: The dimension of a reaction

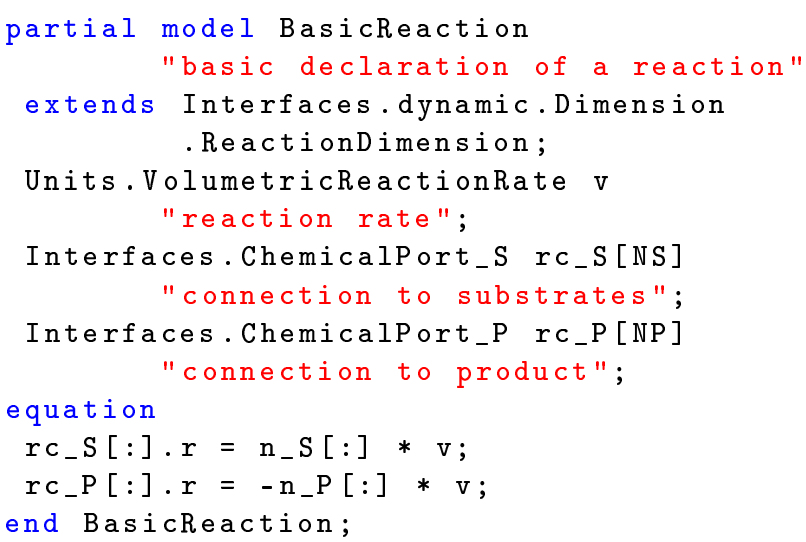

Specification of the reaction reversibility is done via the related classes OneWayReaction and TwoWayReaction. These classes provide the basic declaration of related kinetic parameters and are directly taken from Biochem. Moreover, two additional abstract classes BasicIrrReaction and BasicRevReaction are introduced in the proposed library for emphasizing type abstractions among implemented kinetics:

Listing 7: Basic reversible reaction

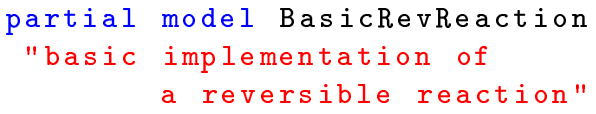




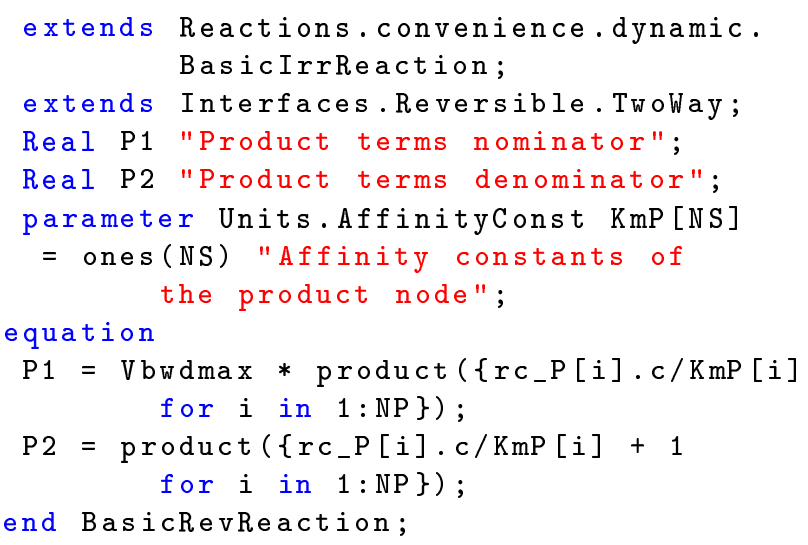

The corresponding classes for specifying the effectors are given by the classes ReactionInhibition and ReactionActivation:

Listing 8: The inhibitors of a reaction

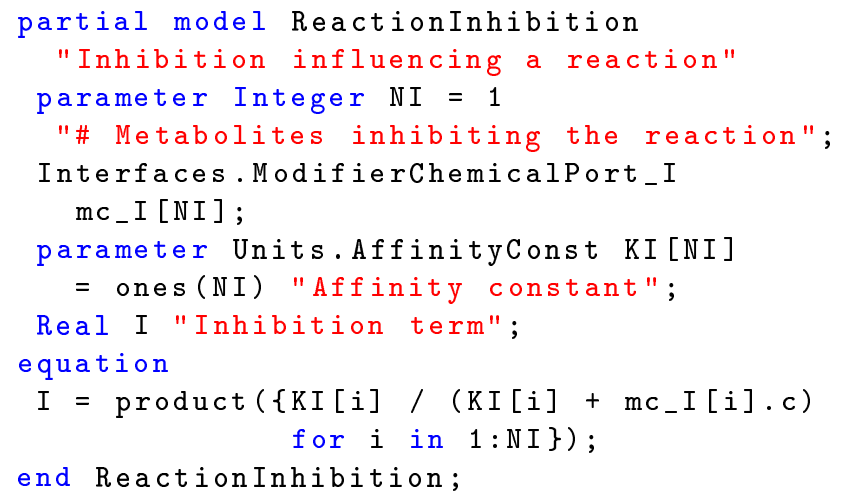

Using these classes, all reaction types of convenience kinetics are realized only with 8 classes. For instance, the implementation of convenience kinetics for reversible inhibited reactions with arbitrary number of reactant substrates, products and inhibitors is given as follows:

Listing 9: Kinetic for reversible inhibited reaction

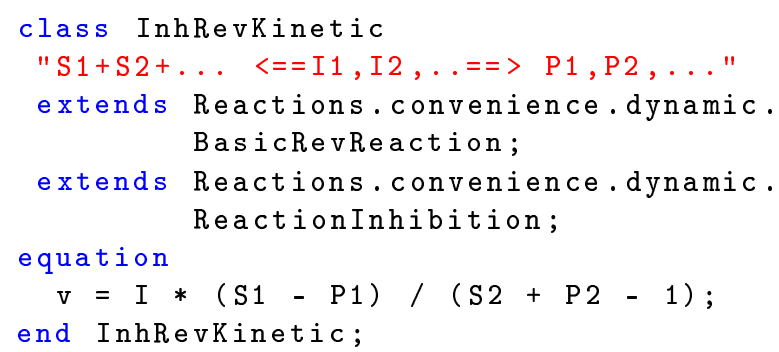

Realistic biochemical reaction networks can be realized using only these 8 classes.

\subsection{2 static}

This subpackage is more or less a straightforward implementation of the Biochem guidelines except in some details. It provides many components for describing enzyme kinetics with fixed number of substrates, products and modifiers via a static number of connectors. The implementation is done along many levels via the following abstract classes:

1. The classes Reaction $1 S$, Reaction $2 S, \ldots$, Reaction1P, Reaction2P, ... , Reaction1I, Reaction1A etc. provide the basic icons for reactions with specific number of connectors to substrates, products, etc.

2. The abstract classes BasicIrrReaction1S1P, BasicIrrReaction $2 S 1 P, \ldots$ etc. provide basic implementation for kinetic terms of irreversible and reversible reactions with specific number of reactants. Similarly, the classes BasicReaction1I, BasicReaction1I provide basic implementation for kinetics terms of modified reactions.

3. The actual kinetics are realized within $I r$ rKinetic1S1P, IrrKinetic1S2P, ... , IrrKinetic $1 S 1 P 1 I, \ldots$ etc. by extending and specializing the abstract classes.

Using this way, many components need to be provided. For instance, by realizing reactions with two substrates and two products together with one modifier at maximum, there are 2 (substrates) $\times 2$ (products) $\times$ 2 (reversibility) $\times 3$ (effectors) $=24$ components that need to be provided. By three substrates and three products with two modifiers at maximum, about $9 \times$ $2 \times 6=108$ components need to be provided.

\subsection{The Derivatives subpackage}

The Derivatives subpackage contains an extended copy of the whole library with identical structure of subpackages, interfaces and components. Each component has additional declaration and equations for computing parameter sensitivities. The equations are computed using algorithmic differentiation techniques. In this work, new novel $\mathrm{AD}$ techniques especially optimized for equation-based languages are employed. For any model using the library typically corresponding to a DAE system of the form:

$$
F(\dot{x}, x, p, t)=0 \quad, \quad x\left(t_{0}\right)=x_{0}(p)
$$

where $x(t) \in R^{n}$ and $p \in R^{m}$ represent state variables and model parameters, respectively, importing the types within the Derivatives subpackage lets the underlying model of eq. (10) get extended with the the 


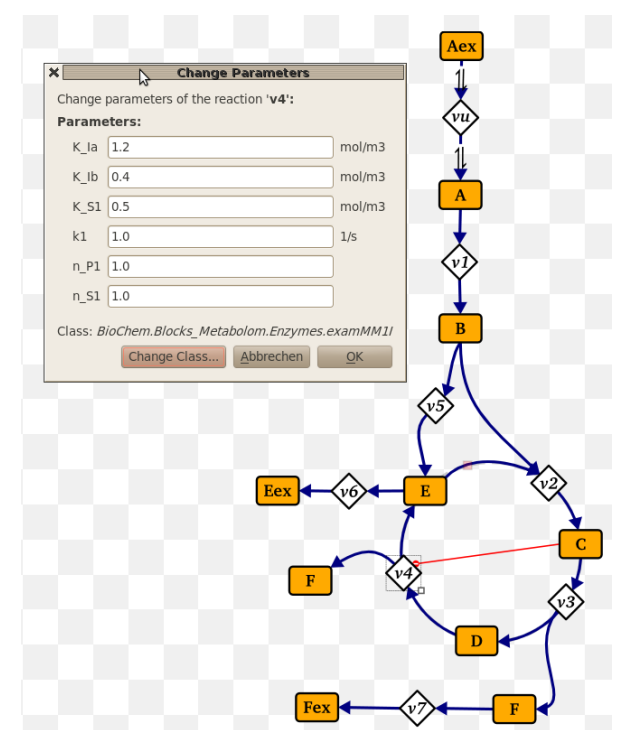

Figure 4: Omix: a highly-specialized graphical editor for biochemical networks

corresponding sensitivity subsystems:

$$
\begin{gathered}
{\left[F_{\dot{x}} \dot{s}_{i}+F_{x} s_{i}+F_{p_{i}}\right] J_{p}=0 \quad, \quad s_{i}\left(t_{0}\right)=\frac{\partial x_{0}(p)}{\partial p_{i}}} \\
\text { where } \quad s_{i}=\frac{\partial x}{\partial p_{i}} \quad \text { for } \quad i=11,2, \ldots, m
\end{gathered}
$$

and $J_{p} \in R^{m \times r}$ is the input Jacobian specifying the set of active parameters $q \in R^{r}$ w.r.t. which derivatives are sought. The same model simulates the underlying biochemical reaction network together with the derivatives of all variables with respect to the specified input parameters. A usage example is available in the Examples subpackage and is summarized in the following section.

\section{Examples}

The implementation of the biochemical network in figure 1 is demonstrated once with dynamic number of connections and again with static number of connections. With dynamic number of connections, the implementation is assembled as follows:

Listing 10: Implementation of the Spirallus network with parametrized number of connections

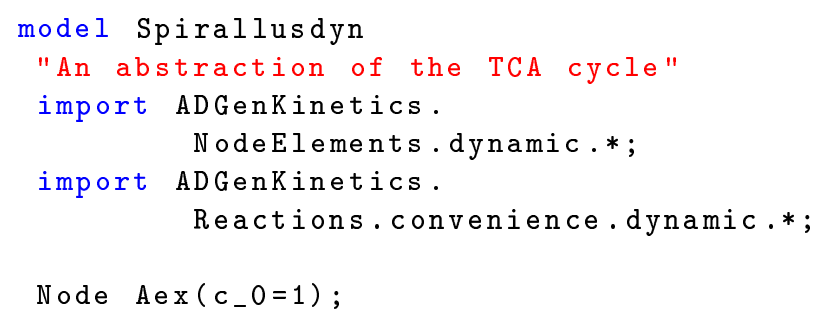

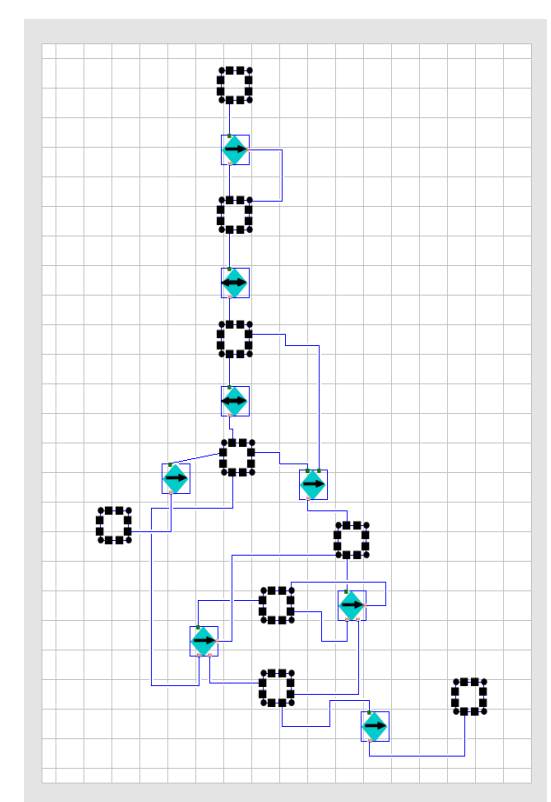

Figure 5: Implementation with the Dymola graphical editor

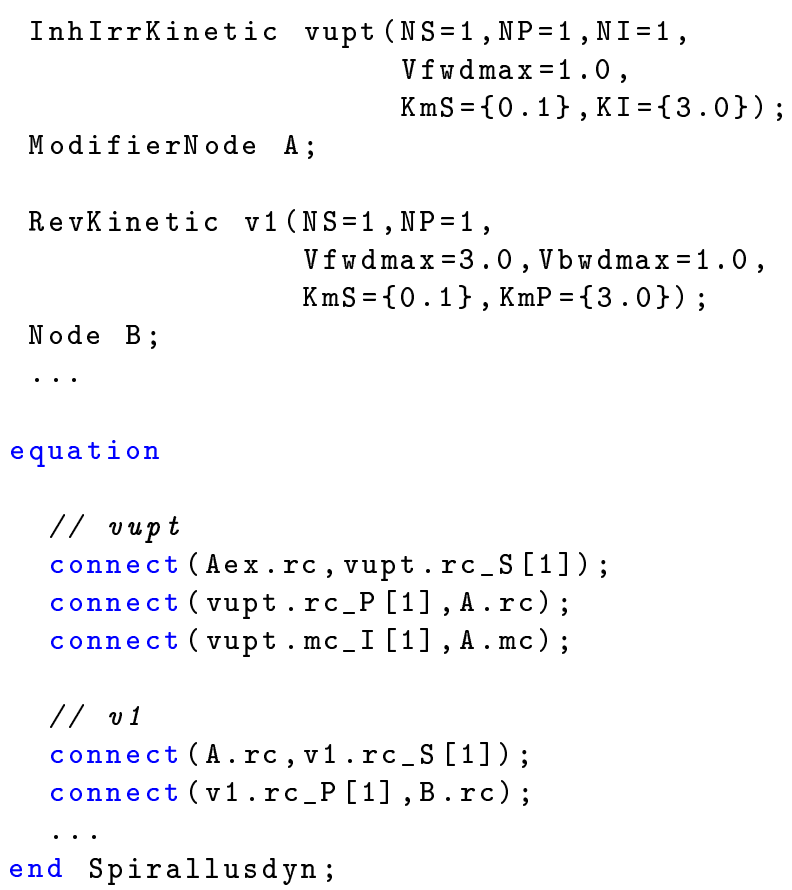

The main disadvantage of this approach is that the implementation is provided only at textual level. Typical modeling environments of Modelica don't currently provide graphical support for parametrized dynamic number of connections yet. Nevertheless, this approach is ideally relevant for automatic model generation possibly using specialized graphical editors for biochemical networks. For instance, figure 4 shows a snap shot of Omix [2] a general-purpose editor for constructing, editing and visualizing biochemical networks in a semi-automatic manner. 


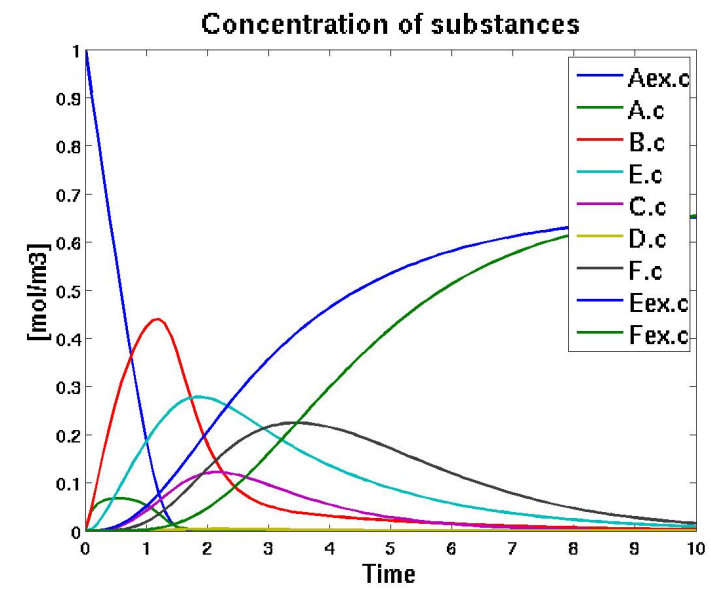

Figure 6: Concentration of the substances

Omix is enhanced with a plugin for parsing and generating Modelica models [13]. The tool employs Open Modelica Compiler (OMC) for parsing a Modelica library for biochemical network modeling and identifying existing types of kinetics and nodes. Then OMC is again used for automatically generating the corresponding models that can be then simulated using typical Modelica simulation environments as described in [3] in a very similar manner to the tool provided in [4]. The presented library would be ideal for such a tool or any other SBML-based graphical editor using very similar concepts.

With the static components of fixed number of connections, biochemical network models can be directly assembled with common Modelica simulation environments. For instance, figure 5 provides the implementation of the network model using Dymola. Figures 6 and 7 demonstrate the simulation results of the concentration of chemical substances and the reaction rates of reactions of the Spirallus network, respectively. Using the subpackage Derivatives, parameter sensitivities can be computed in a straight forward way. For the Spirallus example, this can be done by slightly modifying the declaration part of the code from listing 10 to the following:

Listing 11: Implementation of the dynamics of the Spirallus network together with the parameter sensitivities

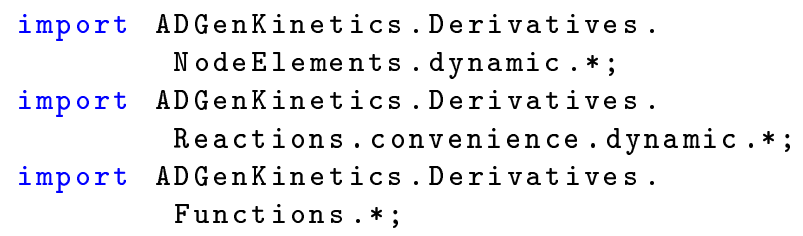

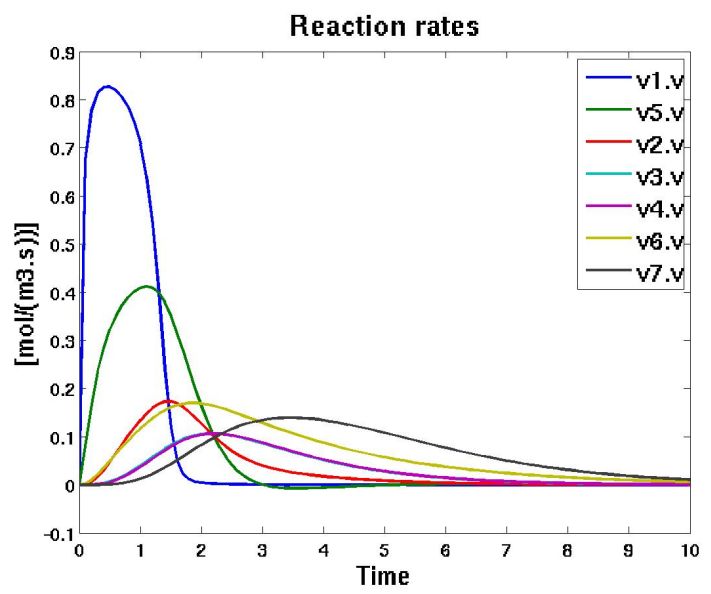

Figure 7: Reaction rates of reactions

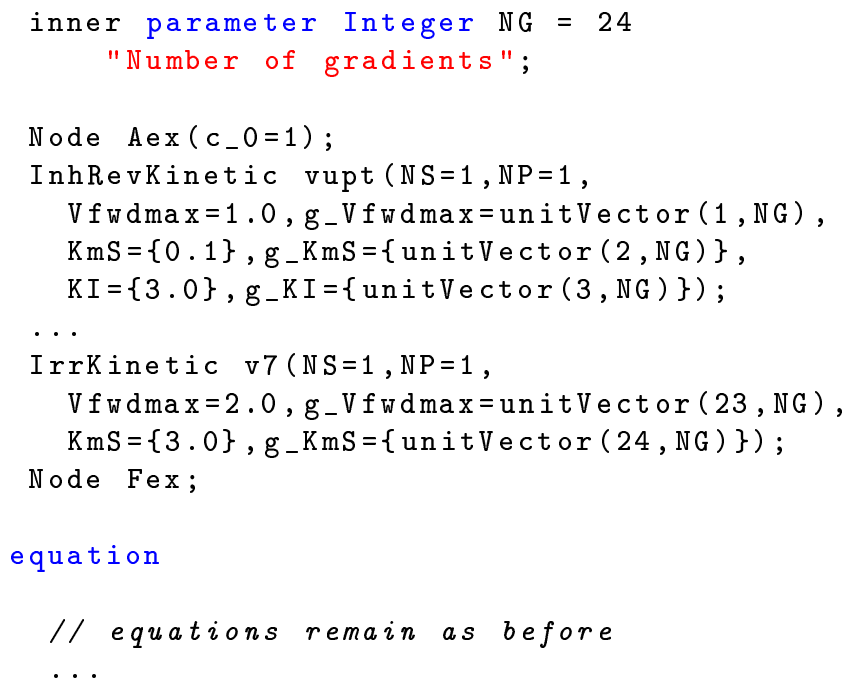

In the last model, the standard types for nodes and reactions are replaced by the extended types within the subpackage Derivatives. An additional unique parameter $N G$ is declared, specifying the number of active parameters w.r.t. which derivatives are sought. Finally, the input gradient of any parameter $p$ is initialized with the help of the function unitVector $(i, N G)$ which returns a unit vector of length $N G$ with the $i$ th component equal to one. In this way, for any variable $v, g_{-} v[i]$ corresponds to $\partial v / \partial p$. For parameters with non-initialized gradients, they simply become inactive. Figure 8 shows the parameter sensitivities of the reaction $v_{7}$ w.r.t. all kinetic parameters.

\section{Outlook}

In this work, a Modelic library for implementing generalized kinetics formats based on justifiable simplification assumptions is provided. With the help of Modelica language constructs, the opportunity of real- 


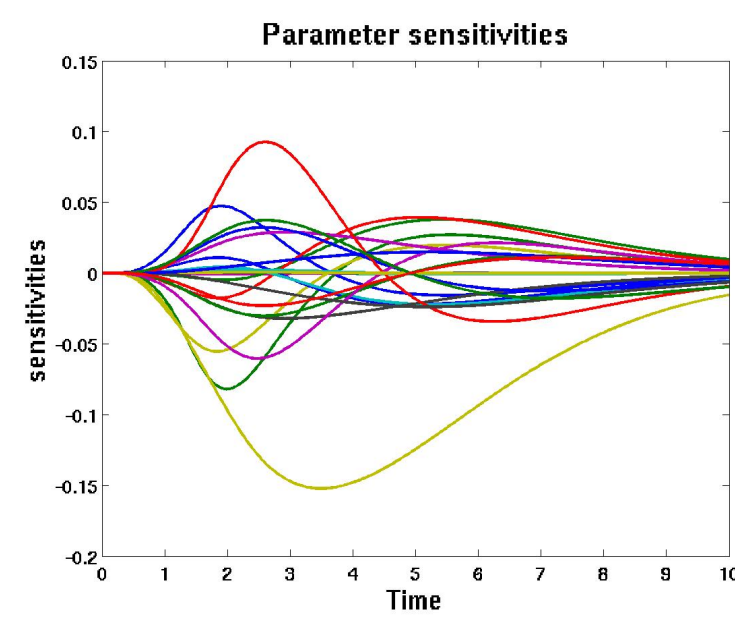

Figure 8: Parameter sensitivities of $v_{7}$

izing real-life applications with few number of components is given. Consequently, the library is especially adequate for tools requiring automatic model generation. Moreover, this library follows the main guidelines of Biochem making it possible to get integrated with other existing implementation. The presented library is the first algorithmically differentiated Modelica library. With minimal additional user efforts, base models additionally simulate parameter sensitivities together with the network dynamics. The underlying novel equation-based $\mathrm{AD}$ techniques which have been especially designed for ADGenKinetics have also the potentials to be employed by other Modelica libraries.

\section{Acknowledgement}

I'd like to acknowledge Dr. Stephan Noack, institute of Bio- and Geosciences, Biotechnology (IBG-1), research centre Jülich, for valuable discussions about enzyme kinetics. His own advanced modeling library was very inspiring for me for understanding the principles of modeling biochemical reaction networks.

\section{References}

[1] H. Bisswanger. Enzyme Kinetics, Principles and Methods. WILEY-VCH Verlag, Weinheim, Germany, 2002.

[2] P. Droste, S. Noack, K. Noh, and W. Wiechert. Customizable visualization of multi-omics data in the context of biochemical networks. In VIZ 2009: The 2nd International Conference on Information Visualisation, Barcelona, Spain, 2009.
[3] A. Elsheikh. Modelica-based computational tools for sensitivity analysis via automatic differentiation. $\mathrm{PhD}$ thesis, submitted to Institute of Computer Science, RWTH Aachen University, Aachen, Germany, 2011.

[4] A. Elsheikh, S. Noack, and W. Wiechert. Sensitivity analysis of Modelica applications via automatic differentiation. In Modelica'2008: The 6th International Modelica Conference, Bielefeld, Germany, 2008.

[5] A. Elsheikh and W. Wiechert. Automatic sensitivity analysis of DAE-systems generated from equation-based modeling languages. In $\mathrm{C}$. $\mathrm{H}$. Bischof, H. M. Bücker, P. D. Hovland, U. Naumann, and J. Utke, editors, Advances in Automatic Differentiation, pages 235-246. Springer, 2008.

[6] A. Griewank. Evaluating Derivatives: Principles and Techniques of Algorithmic Differentiation. Number 19 in Frontiers in Appl. Math. SIAM, Philadelphia, PA, 2000.

[7] F. Hadlich, S. Noack, and W. Wiechert. Translating biochemical network models between different kinetic formats. Metabolic Engineering, 11(2):87 - 100, 2009.

[8] J. J. Heijnen. Approximative kinetic formats used in metabolic network modeling. Biotechnology and Bioengineering, 91(5):534545, 2005.

[9] E. Klipp, R. Herwig, A. Kowald, C. Wierling, and H. Lehrach. Systems Biology in Practice: Concepts, Implementation and Application. Wiley-VCH, 2005.

[10] W. Liebermeister and E. Klipp. Bringing metabolic networks to life: convenience rate law and thermodynamic constraints. Theoretical Biology and Medical Modelling, 2006.

[11] U. Naumann. The art of Differentiating Computer Programs, an Introduction to Algorithmic Differentiation. SIAM, 2012.

[12] E. L. Nilsson and P. Fritzson. A Metabolic Specialization of a General Purpose Modelica Library for Biological and Biochemical Systems. In Proceeding of the 4th International Modelica Conference, Hamburg, Germany, 2005. 
[13] J. Tillack, P. Droste, N. Hackbarth, W. Wiechert, and K. Nöh. Visually-assisted modeling of kinetic metabolic networks - from Omix to Modelica and back. In MATHMOD 2012: The 7th Vienna International Conference on Mathematical Modelling, Vienna, Austria, 2012.

[14] S. A. Wahl. Methoden zur integrierten Analyse metabolischer Netzwerke unter stationären und instationären Bedingungen. $\mathrm{PhD}$ thesis, Research Centre Jülich, Germany, 2007.

[15] W. Wiechert, S. Noack, and A. Elsheikh. Modeling languages for biochemical network simulation: Reaction vs equation based approaches. Advances in Biochemical Engineering / Biotechnology, 2010.

[16] W. Wiechert and R. Takors. Validation of metabolic models: Concepts, tools, and problems. In H. V. Westerhoff and B. Kholodenko, editors, Metabolic Engineering in the Post Genomic Era (Horizon Bioscience). Horizon Scientific Press, 2004. 